\title{
INFLUENCE OF SPATIAL EFFECTS ON COHERENT STRUCTURES FORMATION IN A SUPERSONIC FLOW NEAR A PLATE
}

\section{I. Lipatov and R. Ya. Tugazakov}

Central Aerohydrodynamic Institute (TsAGI)

1 Zhukovsky Str., Zhukovsky, Moscow Region 140180, Russia

\begin{abstract}
Formation of coherent structures in supersonic flow near flat plate with a reflecting strong shock wave is studied. Also, a problem of harmonic wave influencing the flow near plate has been investigated. Similar mechanisms of coherent structures formation in both unsteady problems are found due to secondary instability arising in a flow.
\end{abstract}

\section{INTRODUCTION}

A large number of experimental works investigating vortex structures in threedimensional (3D) unsteady flows of gas were published [1-6]. Numerical modeling of processes of instability in mixing layers accompanied by vortex structures formation was carried out in [7-12]. The mechanisms leading to the cross-flow formation near the plate of finite width under strong falling shock wave were described in $[10,11]$. It was shown that interaction of longitudinal vortices with the transversal vortices may lead to the structures formation similar to the $\Lambda$-structures investigated in subsonic flows [5]. In [8], receptivity of the flow to the entropy disturbances was investigated. As was shown in [12], width and length of the plate play significant role in pressure pulsations level increase.

The purpose of the present article is the cross and longitudinal structures investigation which are formed due to shock wave falling on a plate along with harmonic waves influence. Disturbances amplification coefficients were determined as functions of wavelength and plate width ratio.

In $[9,10]$, investigation was conducted for Mach numbers 6 and for high values of stagnation pressure. Still unresolved was a problem to describe the flows with smaller speeds and for smaller values of Reynolds numbers. For this purpose, in this paper, the flow regime corresponding to Mach number 2 is investigated.

(C) The authors, published by EDP Sciences. This is an Open Access article distributed under the terms of the Creative Commons Attribution License 4.0 (http://creativecommons.org/licenses/by/4.0/). 


\section{PROBLEM FORMULATION}

The flow schemes are presented in Fig. 1 where abcd corresponds to the supersonic flow near plate with a reflected strong shock wave and supersonic flow near the plate influenced by travelling harmonic wave.

In the first case, as a result of shock wave reflection, boundary layer separation takes place with the unsteady vortex in the separated region $[7,9,10]$.

In the second case, harmonic waves lead to the Tolmien-Shlichting instability and separated zones formation having lengthscale comparable with the wavelength.

Wave reflection from side edges of a plate transforms from originally twodimensional (2D) flow to three-dimensional (3D) flow. In both cases, the flow in the wake contains transversal vortices with the parameters depending on the plate width, wavelength, and Mach number.

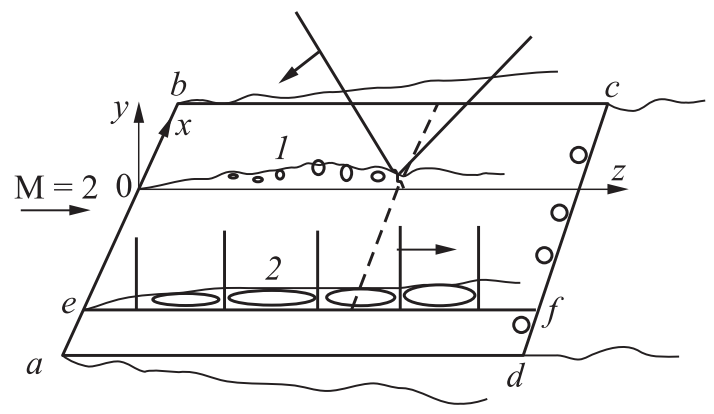

Figure 1 Flow shemes nearby the flat plate: 1 - strong shock wave falling on the plate; and 2 - harmonic wave

Symmetric flow is supposed so that only the flow near the lower plate surface is considered. The numerical results were obtained by means of direct numerical solution of Navier-Stokes equations $[9,10]$. The accuracy of calculations was estimated by the value of the boundary layer thickness. Data obtained coincide with the theoretical results for central longitudinal section. For separated flow region, the calculated results coincided with the experimental results for cross structures thickness [1] and for pressure pulsations frequencies [2] as well.

To get nondimensional results, all geometrical parameters were divided to the plate length, flow parameters were divided to undisturbed pressure and density, and velocity components were divided by undisturbed speed of sound.

The corresponding Reynolds number takes then 1.2-1.5 million length and is equal $(1.2-1.5) \cdot 10^{6}$. The number of grid points is equal to one million. 


\section{RESULTS OF CALCULATIONS OF THE SHOCK WAVE REFLECTION}

Let us compare the results of calculations corresponding to different Mach numbers $(\mathrm{M}=6[10]$ and 2$)$. The pictures of vortex structures for 4 time values (from top to down) in the form of lines of constant density are given in Fig. 2. For $\mathrm{M}=6$, the time interval of fields of density is equal to $d t=4 \mu \mathrm{s}$, and for $\mathrm{M}=2-20 \mu \mathrm{s}$. It may be concluded that for Mach number 2 (see Fig. $2 b$ ), for smaller values of Re number, the size of vortices increases. Larger vortices promote to form new vortices arising in viscous sublayer (2). In Fig. 2, flow
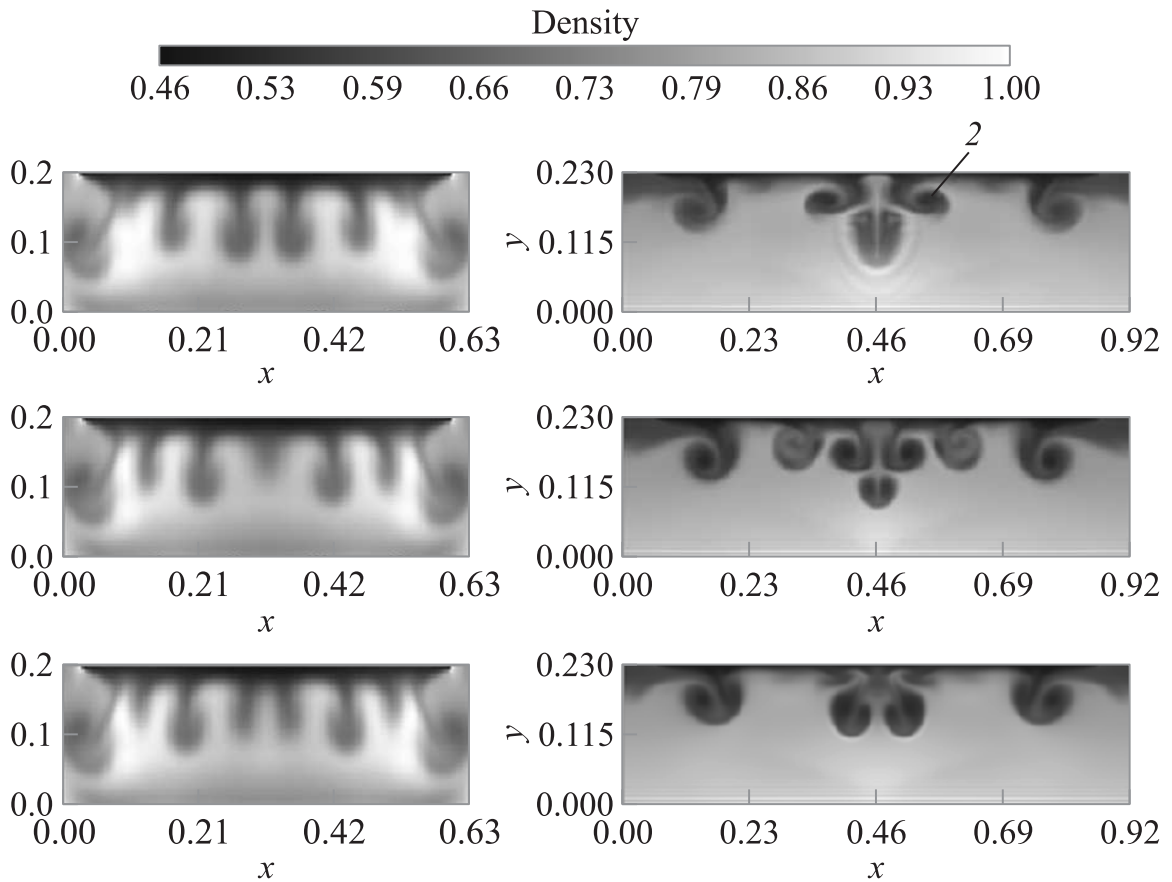

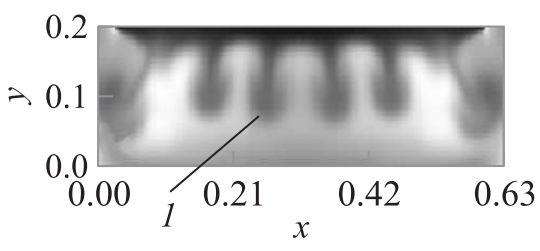

(a)

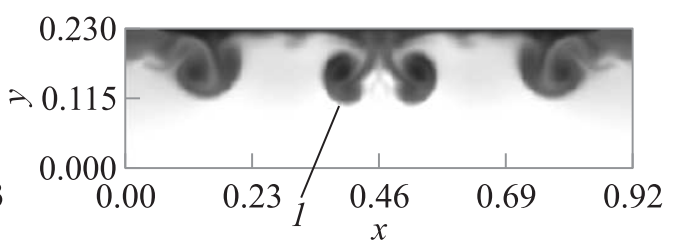

(b)

Figure 2 Vortex structures (1) behind the plate for four moments of time (from top to bottom): (a) $\mathrm{M}=6(z=1.4)$; and $(b) \mathrm{M}=2(z=1.3)$ 

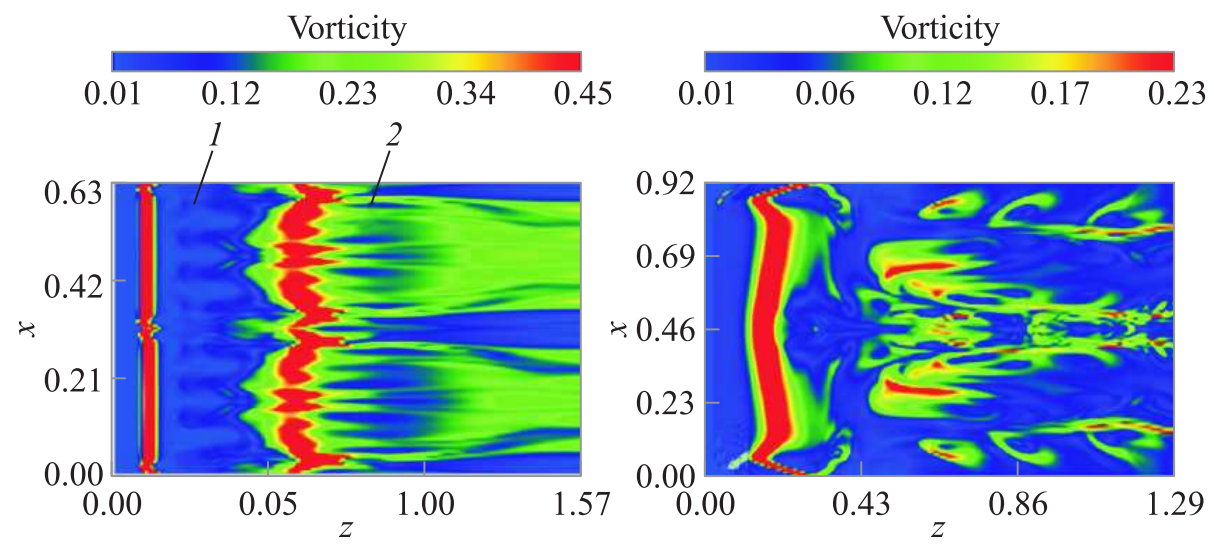

(a)
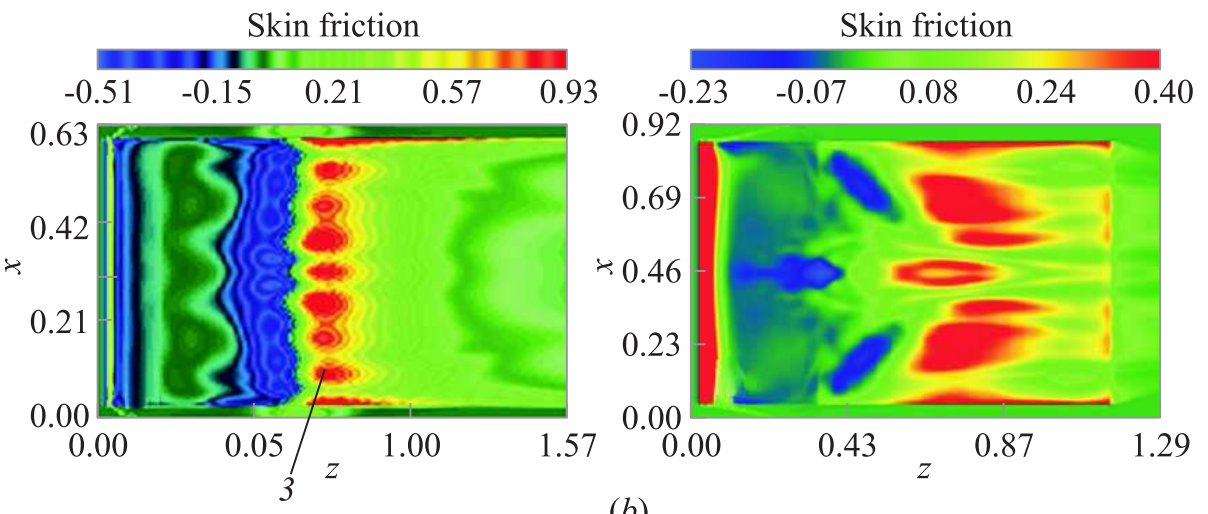

(b)

Figure 3 Vorticity $(a)$ and skin friction distributions (b) on plates $\mathrm{M}=6$ (left column) and 2 (right column): 1 - striky structures; 2 - longitudinal structures; and 3 - large skin friction regions

structures for wider plate are depicted. It may be concluded that flow structure near narrow plate $(\mathrm{M}=2)$ is characterized by two vortices collision arising near side edges of the plate.

Vorticity fields along with the skin friction distributions are depicted in Fig. 3. It may be concluded that for $\mathrm{M}=6$ (see Fig. 3, left column) downstream from the shock wave, streaky structures arize and interact with cross vortices. This process leads to the formation of cross structures consisting of two longitudinal vortices having opposite rotation. In the region of vortices interaction, viscous sublayer arizes leading to the skin friction increase almost on two orders (Fig. $3 b$, left column). 
In the flow near plate for $\mathrm{M}=2$, flow picture for vorticity field and skin friction distribution differ from the data obtained for larger Mach number. Here, one can see stronger viscosity influence along with the change of the shock wave amplitude.

Thus, comparison of the results obtained for two sources of disturbances leads to the conclusion about similarity of flow fields with the flow velocity as a main factor influencing the vortices parameters.

\section{RESULTS OF CALCULATIONS FOR THE TRAVELLING WAVE INFLUENCE}

Let us consider a travelling of sound wave and influencing supersonic flow near flat plate with $\mathrm{M}=2$. This problem contains additional significant parameter wavelength. It was found that nondimensional parameter proportional to the wavelength to the plate width ratio strongly influences the wavelength amplitude: it can diminish or grow. It was investigated the flow near the plate having the length of $2.5-3 \mathrm{~cm}$ with the wavelength $1-2.5 \mathrm{~mm}$. Frequency of waves changed in the range of $25-100 \mathrm{kHz}$. The calculations with the amplitude of the incident wave $\approx 1 \%$ of the pressure in the unperturbed gas $P_{0}$ are presented. In Fig. 4, change of a $2 \mathrm{D}$ initial profile of a wave near the plate having unity length is presented. From distribution of pressure on a plate (see Fig. 4, at $y=0)$, it is visible that on the plate border (the light line), there is a reflection of disturbances which leads to a wave bend in the cross direction and forms a number of secondary waves. As a result, the flow in the boundary layer becomes $3 \mathrm{D}$. As a result, the amplitude of pulsations nearby the trailing edge considerably diminishes. The change of an initial profile in longitudinal central section is shown in Fig. 4, Tollmin-Shlichting waves formation can be seen.

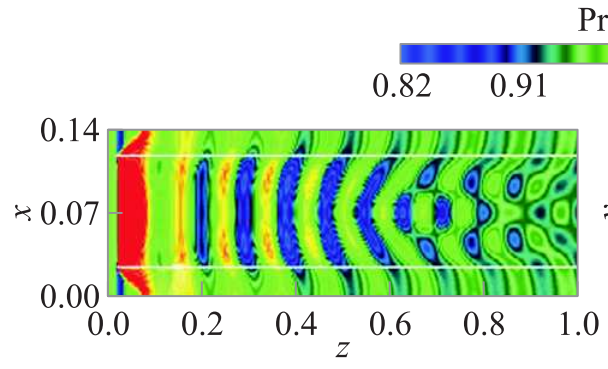

(a)
Pressure
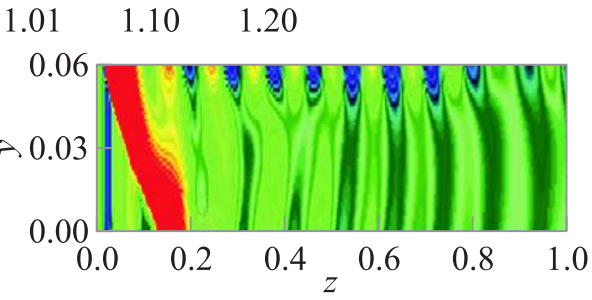

(b)

Figure 4 Pressure field of harmonic wave in the flow on a plate $(a)$ and in longitudinal central cross section $(b)$ 
The results of travelling wave influence on the wake flow downstream from the plate are presented in Figs. 5-7. In particular, for waves with short wavelength $\lambda_{1} \approx 1 \mathrm{~mm}$, the pressure distribution on a plate (at $y=0$ ) is depicted in Fig. $5 a$. It may be concluded that on a narrow plate already at distance $z \approx 0.2$, originally, 2D wave flow turns to be $3 \mathrm{D}$. Vortices along the plate are visible. Downstream from the plate, the strong expansion wave is formed providing gas acceleration coming from the boundary layer region. Pressure and density fields

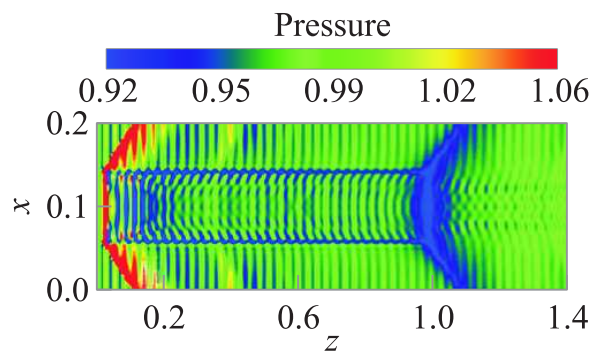

(a)

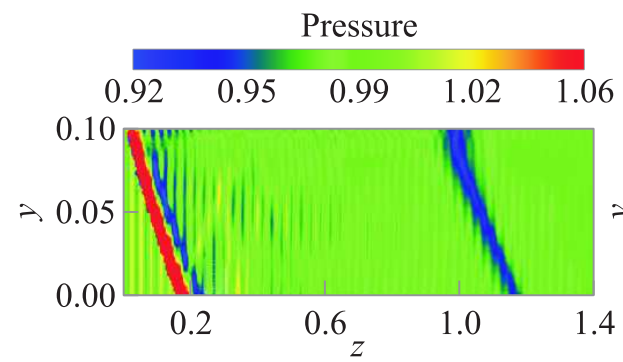

(b)

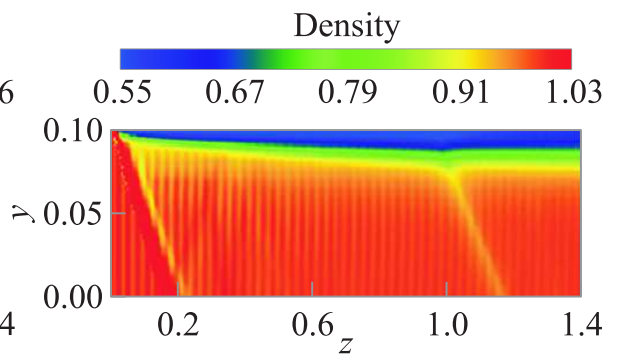

(c)

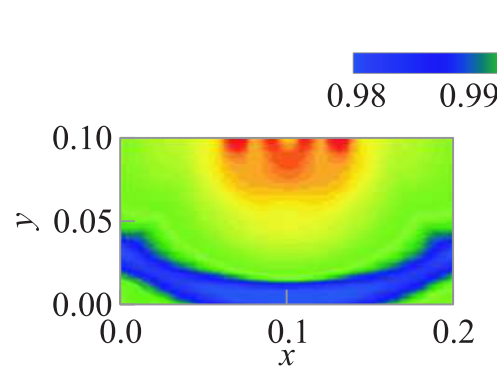

(d)

Vorticity

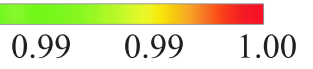

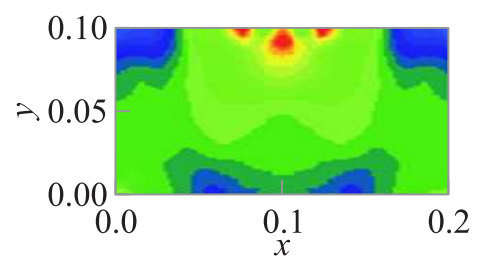

(e)

Figure 5 Flow parameter distributions in the case of flow past for the finite-length plate: $(a)$ pressure on the plate; $(b)$ and $(c)$ pressure and density in the central longitudinal section of the computational domain; $(d)$ and $(e)$ vortex structures in the transverse sections $z=1.17$ and 1.4 , respectively 


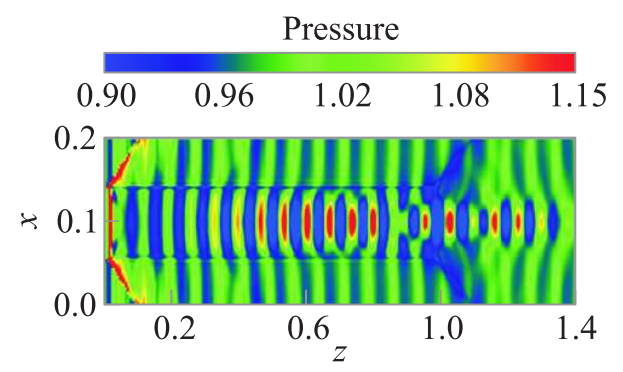

(a)

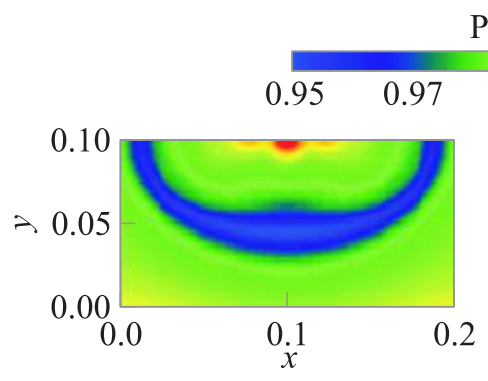

(b)

Pressure
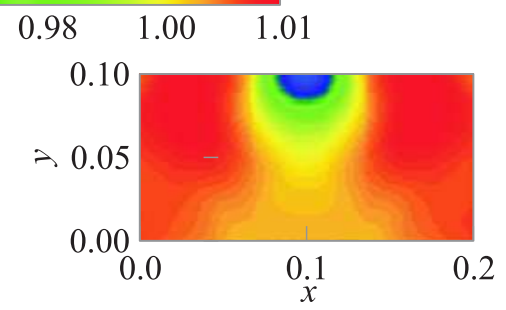

(c)

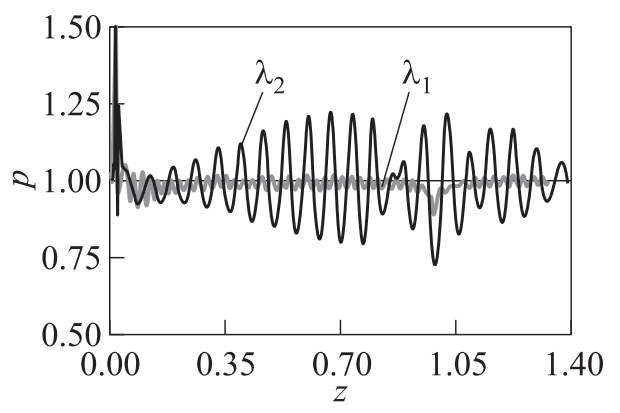

(d)

Figure 6 Pressure on the plate $(a)$ and in the transverse sections of $z=1.1(b)$ and $1.4(c)$ in the case of the doubled wavelength of the oncoming wave and comparison of the pressures $(d)$ on the plate (in the central longitudinal section) for two wavelengths

in longitudinal section of the flow are presented in Figs. $5 b$ and $5 c$. The behavior of the vortex structures which are formed behind a plate in cross sections of $z=1.17$ and 1.4 are shown in Figs. $5 d$ and $5 e$. It may be concluded that in the abovementioned cross sections, 3 or 4 vortices are formed. In cross section $z=1$, five vortices exist and it may be concluded that for larger $z$ values, number of vortices diminishes. 


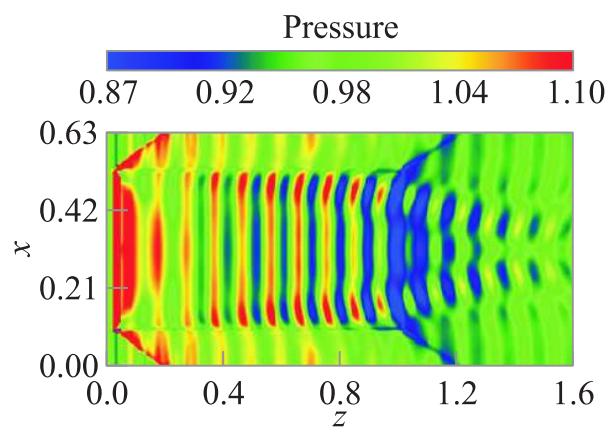

(a)

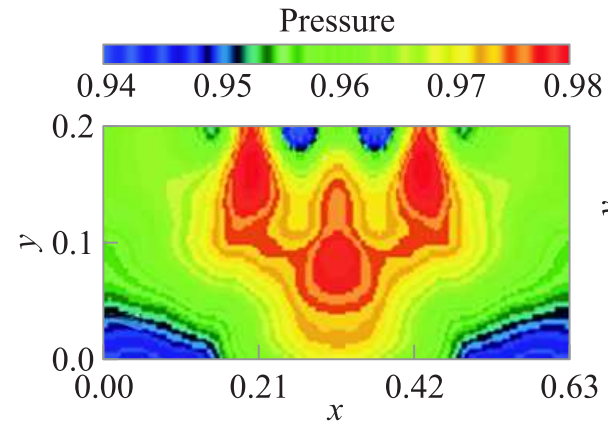

(b)

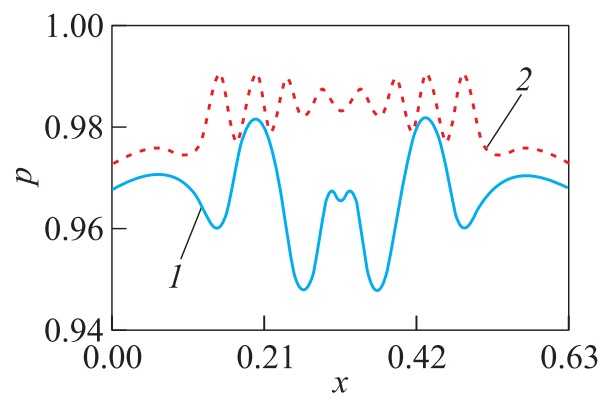

(d)

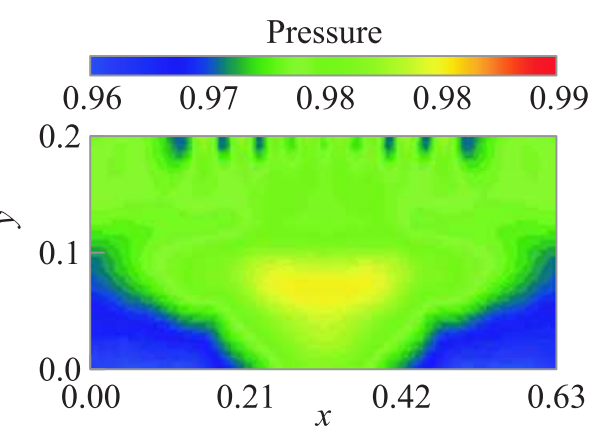

(c)

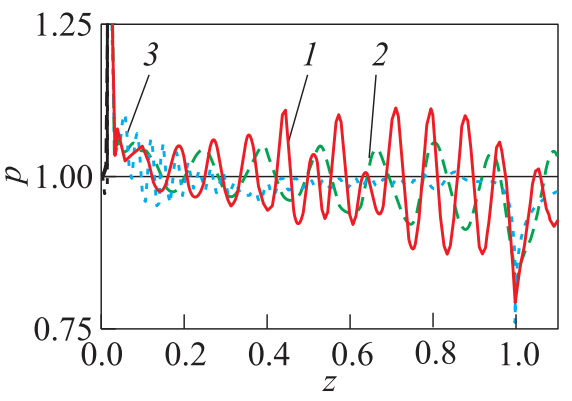

(e)

Figure 7 Vortex structure on the wide plate: $(a)$ wave structure of the flow on the plate; $(b)$ and $(c)$ vortex structures in the transverse sections of the wake $(z=1.5)$ for long and short waves; $(d)$ comparison of the wake pressures $(y=0$ and $z=1.5)$ for the long (1) and short waves (2); and (e) pressure fluctuation growth on the plate for the long (1 and 2$)$ and short waves (3) 
To show influence of the wavelength on the flow, the calculations were made for larger wavelength $\lambda_{2} \approx 2.3 \mathrm{~mm}$. From the results presented in Fig. 6 , it may be concluded that the travelling wave on the plate is deformed as well but does not separate into series new waves, that is, for such plate when its width of an order of length of the travelling wave, final destruction of an initial wave does not occur. Therefore, in cross sections behind a plate $(z=1.1$ and 1.4$)$, longitudinal vortices are not visible because their intensity is insignificant. Such difference in flow structures for flows with different wavelengths influences the flow parameters. Especially, it is confirmed by the pressure distributions on the plate in longitudinal central section presented in Fig. 6. Here, it is shown that amplitude of waves with a small length (curve 1 ) nearby plate leading edge slightly grows up and then, after waves destruction $(z \approx 0.2)$, considerably decreases. Amplitude of waves with the doubled length (curve 2) increases more in comparison with initial amplitude. Wavelength $\lambda \approx 2 \delta$ is also equal in this option to the plate width $h$, having boundary layer thickness $\delta=0.9 \mathrm{~mm}$. In Fig. $7 a$ where pressure field is presented for the wide plate and for the travelling wave with the wavelength $\lambda_{2}$, it is visible that the wave does not break up to separate waves. Dependence of number of vortex structures on long and short waves is presented in Figs. $7 b$ and $7 c$ for $z=1.5$. It is visible that number of vortex structures is in inverse proportion to the wavelength which follows from the pressure distribution in the wake (Fig. $7 d$ ). Dependence of amplitude of pulsations on a wide plate from wavelength is shown in Fig. $7 e$. Just as on a narrow plate for short waves (3) at the leading edge of plate, there is a destruction of a wave and amplitude of pulsations falls. For doubling wavelength $\lambda_{2}=2 \lambda_{1}$, there is a sharp growth of amplitude of a wave (1). However, at further increase in wavelength twice $\left(\lambda_{3}=2 \lambda_{2}\right)$, its amplitude falls almost twice $(2)$, that is, there is a certain wavelength providing the maximum strengthening of disturbances. Comparison of pressure distributions on the narrow (Fig. 6d) and on the wide plates (Fig. 7e) shows that strengthening of amplitude of waves is twice more on a narrow plate. It means that strengthening of disturbances on a plate depends both on the plate width and on the wavelength of the travelling wave.

\section{THE ANALYSIS OF DEPENDENCE OF AMPLIFICATION OF PRESSURE PULSATIONS ON THE WAVELENGTH}

In Fig. 8, the amplification coefficients $\Delta P_{\max } / \Delta P_{0}$ are presented that depend on the dimensionless parameter proportional to the wavelength ratio to the boundary layer thickness $\delta$. It may be concluded that the maximum strengthening takes place at wavelength equal to 2 boundary layer thickness. For this case, significant increase in amplitude of travelling wave takes place. Influence of width 


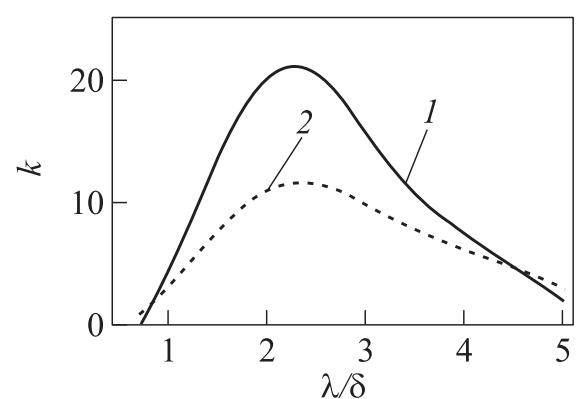

(a)

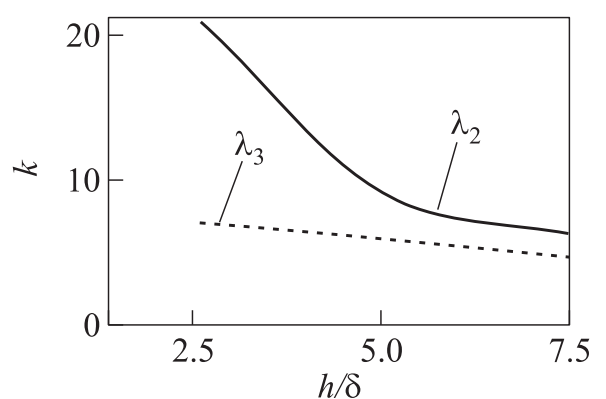

(b)

Figure 8 Wave amplitude amplification coefficients $k=\Delta P_{\max } / \Delta P_{0}:(a)$ dependence on nondimensional wavelength ( 1 and 2 - narrow and wide plates); and ( $b$ ) plate width influence

of a plate on amplification coefficient for two waves with different wavelengths is given in Fig. $8 a$ where curves 1 and 2 show essential distinction in amplification coefficient for narrow and wide plates, that is, for narrow plates when interaction of side vortices is essential.

It is possible to explain effect of strengthening of amplitude by means of waves interaction with the boundary layer flow. When the wave moves along a mixing layer, there is its refraction because of action of a positive and negative phase of a wave. The bend of a mixing layer leads to strengthening of pressure in a positive phase and to reduction in the negative. When wavelength is less than $\delta$, these changes are compensated in the boundary layer, that is, the gain and reduction of pressure extinguish themselves without amplifying, without reaching a plate. In a case when wavelength is of the order of $2 \delta$, compression and expansion waves reach a wall and amplify in amplitude. In the process of movement of the wave along the mixing layer, there is an accumulation of these disturbances that leads to the further growth (reduction) of amplitude of a wave and formation of cross vortices. In fact, a typical Kelvin-Helmgolz instability takes place. As in the problem with a shock reflection on side borders, in the cross direction sound waves exist and the scenario of development of instability is, probably, the same. But here, waves interact in the middle of a plate and are reflected from each other. When wavelength of the waves reflected from the side borders several times is less than a half of width of a plate, collision of phases of compression and expansion occurs not on the plate border but in its central part. In this place, the weak vortex arize. But there is the main longitudinal speed and, as a result of this interaction, the longitudinal vortex is formed. As the length of a cross wave is less than $h / 2$ for several times, some longitudinal vortices of weak intensity which are visible in Figs. 4 and 5 are formed. In the case when the length of a cross wave is of the order 
of $h$, in internal area on a plate, the longitudinal vortices are not formed (see Figs. 6 and $7 a$ ). It should be noted that the analysis of laminar-turbulent transition [8] in 2D formulation is not correct and should take into account the $3 \mathrm{D}$ effects.

\section{CONCLUDING REMARKS}

Generalizing results of experimental and numerical modeling, the known fact is justified. This fact is associated with the secondary flow instability arising due to $3 \mathrm{D}$ effects. It means that any $3 \mathrm{D}$ surface disturbance may lead to the longitudinal vortices and, eventually, to the secondary instability. In particular, in the problem of reflected shock wave weak longitudinal vortices interacting with the lateral vortices can arise and, as a result, cross structures can appear. Gas emission from the boundary layer provides transition to the turbulence. In the second problem associated with the travelling waves influencing the flow near the plate, there is an additional parameter, the wavelength. Depending on the ratio of the wavelength to the plate width, different solutions are realized in the wake of the plate and the intensity of the wave on the plate becomes stronger or weaker. In the case when wavelength is less than the width of the plate, as a result of interaction of the travelling $2 \mathrm{D}$ wave and vortices arising on lateral parts of the plate, the travelling wave becomes $3 \mathrm{D}$. When wavelength is comparable or more than the width of the plate, vertical structure does not arise. The maximum amplification of travelling wave corresponds to narrow plates and waves having wavelength larger than the width of the plate.

\section{ACKNOWLEDGMENTS}

The work is performed with a partial financial support fot the Russian Foundation of Basic Research (Grant No. 14-01-00848).

\section{REFERENCES}

1. Brazhko, V. N. 1979. Periodicheskaya struktura techeniya i teploperedachi v oblasti prisoedineniya sverkhzvukovykh potokov [Periodic structure of flow and heat transfer in the field of accession of a supersonic stream]. Uchenye zapiski TsAGI [TsAGI Scientific Notes] 10(2):113-118.

2. Glotov, G. F. 1995. Peculiarities of formation and development of recirculation-flow zones in shear layers of supersonic flows. J. Appl. Mech. Tech. Phy. 36(5):666-674. 
3. Gaponov, S. A., and A. A. Maslov. 1980. Razvitie vozmushcheniy v szhimaemykh potokakh [The development of perturbations in compressible flows]. Novosibirsk: Nauka. 143 p.

4. Gutmark, E. J., K. S. Schadow, and K. A. Yu. 1995. Mixing enhancement in supersonic free shear flows. Annu. Rev. Fluid Mech. 245:375-417.

5. Kozlov, V. V., G. R. Grek, L. L. Lofdahl, V. G. Chernorai, and M. V. Litvinenko. 2002. Role of localized streamwise structures in the process of transition to turbulence in boundary layers and jets (review). J. Appl. Mech. Tech. Phy. 43(2): $224-236$.

6. Babinsky, H., and J. K. Harvey. 2011. Shock-wave boundary-layer interactions. New York, NY: Cambridge University Press. 461 p.

7. Pirozzoli, S., and F. Grasso. 2004. Direct numerical simulations of isotropic compressible turbulence: Influence of compressibility on dynamics and structures. Phys. Fluids 16:4386.

8. Ryzhov, A. A., and V. G. Sudakov. 2012. Numerical modeling of the receptivity of a supersonic boundary layer to entropy disturbances. Fluid Dyn. 47(3):338-345.

9. Mazo, A.B., E. I. Kalinin, and V. M. Molochnikov. 2013. Eksperimental'noe i chislennoe issledovanie laminarno-turbulentnogo perekhoda za prepyatstviem $\mathrm{v}$ kanale [Experimental and numerical investigation of a laminar-turbulent transition behind an obstacle in a channel]. Conference (International) "Turbulence and Wave Processes" Dedicated to the Centenary of Mikhail D. Millionshchikov Abstracts. Moscow: INTUIT.RU Ltd. 139-141.

10. Lipatov, I. I., and R. Ya. Tugazakov. 2013. Mekhanizm obrazovaniya pul'satsiy davleniya pri padenii udarnoy volny na pogranichnyy sloy [The mechanism of formation of pressure pulsations with the impact of a shock wave on the boundary layer]. Uchenye zapiski TsAGI [TsAGI Scientific Notes] 44(1):62-74.

11. Lipatov, I. I., and R. Ya. Tugazakov. 2014. Mechanism of transverse structure formation in supersonic flow past a body. Fluid Dyn. 49(5):681-687.

12. Golubev, A. Y., and B. M. Efimtsov. 2015. Distinctive features of the pressure fluctuation fields in the vicinities of bulges. Fluid Dyn. 50(1):50-60. 